ANNALES

POLONICI MATHEMATICI

$91.1(2007)$

\title{
Growth of coefficients of universal Dirichlet series
}

\author{
by O. Demanze and A. Mouze (Lille)
}

\begin{abstract}
We study universal Dirichlet series with respect to overconvergence, which are absolutely convergent in the right half of the complex plane. In particular we obtain estimates on the growth of their coefficients. We can then compare several classes of universal Dirichlet series.
\end{abstract}

1. Introduction. Let $f(s)=\sum_{n \geq 1} a_{n} n^{-s}$ be a Dirichlet series and let $\sigma_{a}(f)$ be its abscissa of absolute convergence, defined by

$$
\sigma_{a}(f)=\inf \left\{\sigma \in \mathbb{R}: \sum_{n \geq 1}\left|a_{n}\right| n^{-\sigma} \text { converges }\right\} .
$$

We define $\left\|\sum_{n \geq 1} a_{n} n^{-s}\right\|_{\sigma}=\sum_{n \geq 1}\left|a_{n}\right| n^{-\sigma} \in[0, \infty]$ for all $\sigma \in \mathbb{R}$. We also define the abscissa of convergence $\sigma(f)=\inf \left\{\sigma \in \mathbb{R}: \sum_{n \geq 1} a_{n} n^{-\sigma}\right.$ converges $\}$. If $f$ is given by a finite sum as above, then we say that $f$ is a Dirichlet polynomial. We denote the $p$ th partial sum of $f$ by $S_{p}(f)=$ $\sum_{n=1}^{p} a_{n} n^{-s}$. Let $\mathbb{C}_{+}$be the half-plane of complex numbers with strictly positive real part. We denote by $\mathcal{D}_{\mathrm{a}}\left(\mathbb{C}_{+}\right)$the set of Dirichlet series which are absolutely convergent on $\mathbb{C}_{+}$. This space, endowed with the topology given by the family of seminorms $\|\cdot\|_{\sigma}$, is a Fréchet space. In the following, we fix a strictly decreasing sequence $\widetilde{\sigma}=\left(\sigma_{k}\right)_{k \geq 0}$ of real numbers which converges to 0 .

Definition 1.1. Let $K$ be a compact set included in $\mathbb{C}$. This set is admissible for Dirichlet series if $\mathbb{C} \backslash K$ is connected and $K$ can be written as $K=K_{1} \cup \cdots \cup K_{d}$ with each $K_{i}$ contained in a strip $S_{i}=\left\{z \in \mathbb{C}: a_{i} \leq\right.$ $\left.\Re(z) \leq b_{i}\right\}$ with $b_{i}-a_{i}<1 / 2$ ( $\Re$ means the real part), the strips $S_{i}$ being disjoint.

We denote by $\mathbb{C}_{-}$the left half-plane $\{s \in \mathbb{C}: \Re(s)<0\}$. We can now give the version of Mergelyan's theorem for Dirichlet series in $\mathcal{D}_{\mathrm{a}}\left(\mathbb{C}_{+}\right)$.

2000 Mathematics Subject Classification: 30B50, 41A28, 30B10, 46B25.

Key words and phrases: Dirichlet series, universality, simultaneous approximation. 
Theorem $1.2([3])$. Let $K \subset \overline{\mathbb{C}}_{-}$be a compact set admissible for Dirichlet series, $f$ be a Dirichlet series in $\mathcal{D}_{\mathrm{a}}\left(\mathbb{C}_{+}\right), g$ be a continuous function on $K$ which is analytic in $\stackrel{\circ}{K}$ and $\sigma, \varepsilon$ be two positive real numbers. Then there exists a Dirichlet polynomial $h$ such that

$$
\sup _{z \in K}|h(z)-g(z)|<\varepsilon \text { and }\|h-f\|_{\sigma}<\varepsilon .
$$

We also define the following sets $\mathcal{W}_{\mathrm{a}}$ and $\mathcal{W}_{1}$ of universal Dirichlet series from $\mathcal{D}_{\mathrm{a}}\left(\mathbb{C}_{+}\right)$. The set $\mathcal{W}_{\mathrm{a}}$ has been introduced in [3].

Definition 1.3. We denote by $\mathcal{W}_{\text {a }}$ the set of all Dirichlet series $h \in$ $\mathcal{D}_{\mathrm{a}}\left(\mathbb{C}_{+}\right)$satisfying: for every admissible compact set $K \subset \overline{\mathbb{C}}_{-}$, and every function $g$, continuous on $K$ and analytic in $\stackrel{\circ}{K}$, there exists a sequence $\left(\lambda_{n}\right)_{n \geq 0}$ of integers such that

$$
\sup _{z \in K}\left|S_{\lambda_{n}}(h)(z)-g(z)\right| \underset{n \rightarrow \infty}{\longrightarrow} 0 .
$$

It is well-known that $\mathcal{W}_{\mathrm{a}}$ is a dense $G_{\delta}$ set [3].

Definition 1.4. We denote by $\mathcal{W}_{1}$ the set of all Dirichlet series $h \in$ $\mathcal{D}_{\mathrm{a}}\left(\mathbb{C}_{+}\right)$satisfying: for every admissible compact set $K \subset \mathbb{C}_{-}$, and every function $g$, continuous on $K$ and analytic in $\stackrel{\circ}{K}$, there exists a sequence $\left(\lambda_{n}\right)_{n \geq 0}$ of integers such that

$$
\sup _{z \in K}\left|S_{\lambda_{n}}(h)(z)-g(z)\right| \underset{n \rightarrow \infty}{\longrightarrow} 0 .
$$

The set $\mathcal{W}_{1}$ differs from $\mathcal{W}_{\mathrm{a}}$ because the intersection of the compact sets $K$ with the imaginary axis is now empty. Obviously by using similar methods one can show that $\mathcal{W}_{1}$ is also a dense $G_{\delta}$ set and $\mathcal{W}_{\mathrm{a}} \subset \mathcal{W}_{1}$. These sets are similar to the sets of universal Taylor series defined in [8], [7] respectively. We refer the reader to [6] for a survey and similar results. For other universal Dirichlet series we refer to [5].

In this paper, we first obtain, as in the analytic case [7], estimates on the growth of coefficients of universal Dirichlet series in the sense of $\mathcal{W}_{\mathrm{a}}$ (Theorem 2.2).

Theorem. Let $\sum_{n=1}^{\infty} a_{n} n^{-s}$ be a Dirichlet series in $\mathcal{W}_{\mathrm{a}}$. Let $\left(b_{n}\right)_{n \in \mathbb{N}}$ be a decreasing sequence such that $\sum_{n=2}^{\infty} b_{n} / n \log (n)<\infty$. Then

$$
\limsup _{n \in \mathbb{N}^{*}} \frac{n\left|a_{n}\right|}{e^{\sqrt{b_{n} \log (n)}}}=\infty .
$$

In Section 3 we prove a decomposition theorem with estimates on the coefficients for all series of $\mathcal{D}_{a}\left(\mathbb{C}_{+}\right)$(Theorem 3.6).

Theorem. Let $f=\sum_{n \geq 1} d_{n} n^{-s}$ be a Dirichlet series in $\mathcal{D}_{\mathrm{a}}\left(\mathbb{C}_{+}\right)$. Then there exist $g_{1}=\sum_{n \geq 1} a_{n} n^{-s}$ and $g_{2}=\sum_{n \geq 1} b_{n} n^{-s}$ in $\mathcal{W}_{1}$ such that $f=$ 
$g_{1}+g_{2}$ on $\mathbb{C}_{+}$with

$$
\limsup _{n \in \mathbb{N}^{*}} n\left|a_{n}\right|=\limsup _{n \in \mathbb{N}^{*}} n\left|b_{n}\right|=\limsup _{n \in \mathbb{N}^{*}} n\left|d_{n}\right| .
$$

This is a version of Theorem 5.1 from [7] for Dirichlet series. As a consequence, we conclude that $\mathcal{W}_{1} \neq \mathcal{W}_{\mathrm{a}}$.

Finally, in the universal set $\mathcal{W}_{1}$, a natural question is whether some universal Dirichlet series converge and are continuous on the imaginary axis. A similar property is true for analytic functions on the unit disk (see [7]). To prove this, A. Melas, V. Nestoridis and I. Papadoperakis study universality in the Banach space $A(\mathbb{D})$ of analytic functions on $\mathbb{D}$, continuous on the torus $\mathbb{T}$. The universal Taylor series $\sum_{n \geq 0} b_{n} z^{n}$ with $\sum_{n \geq 0}\left|b_{n}\right|<\infty$ were first investigated by L. Tomm and R. Trautner [10]. To obtain a result on universal Dirichlet series in Section 4, we study universality in the WienerDirichlet algebra of Dirichlet series $\sum_{n>0} a_{n} n^{-z}$ satisfying $\sum_{n \geq 0}\left|a_{n}\right|<\infty$. We then prove the existence of universal Dirichlet series which are continuous on the imaginary axis. Notice that Theorem 4.2 has been independently obtained by V. Nestoridis and C. Papadimitropoulos in [9] with a different approach.

2. Some properties of $\mathcal{W}_{\mathrm{a}}$. In this section, we study the growth of the coefficients of universal Dirichlet series in $\mathcal{W}_{\mathrm{a}}$. Note that such series converge nowhere on the imaginary axis. Taking as $K$ a singleton $\left\{i t_{0}\right\}$ with $t_{0} \in \mathbb{R}$, we see that every universal Dirichlet series diverges at every point of the imaginary axis. Hence its abscissas of convergence and of absolute convergence are both equal to 0 . We obtain a more precise result on the asymptotic behavior of the coefficients.

Lemma 2.1. Let $\sum_{n=1}^{\infty} a_{n} n^{-s}$ be a Dirichlet series in $\mathcal{W}_{\mathrm{a}}$. Let $\left(\varepsilon_{n}\right)_{n \in \mathbb{N}}$ be a decreasing sequence of positive real numbers such that $\sum_{n=2}^{\infty} \varepsilon_{n} / n \log (n)$ $<\infty$. Then

$$
\sum_{n=2}^{\infty} \frac{\left|a_{n}\right|}{e^{\sqrt{\varepsilon_{n} \log (n)}}}=\infty .
$$

Proof. We set $\delta_{n}=e \varepsilon_{n}$ for all $n \in \mathbb{N}$. There exists an $n_{0} \in \mathbb{N}$ such that $\sum_{\text {by }}^{\infty}{ }_{n=n_{0}} \delta_{n} / n \log (n)<1 / 2$. Let $H_{n}$ be the $2 i \pi$-periodic functions on $i \mathbb{R}$ given

$$
H_{n}(i t)= \begin{cases}\frac{n \log (n)}{\delta_{n}} \pi & \text { for }|t|<\frac{\delta_{n}}{n \log (n)}, \\ 0 & \text { for } \frac{\delta_{n}}{n \log (n)} \leq|t| \leq \pi .\end{cases}
$$


We put $\widehat{f}(m)=(2 \pi)^{-1} \int_{-\pi}^{\pi} f(i t) m^{i t} d t$. An easy calculation gives

$$
\begin{aligned}
\widehat{H}_{n}(1) & =\frac{1}{2 \pi} \int_{-\pi}^{\pi} H_{n}(i t) d t=1, \\
\widehat{H}_{n}(m) & =\frac{\sin \left(\frac{\delta_{n}}{n \log (n)} \log (m)\right)}{\frac{\delta_{n}}{n \log (n)} \log (m)}, \quad m \neq 1 .
\end{aligned}
$$

Let $N \geq n_{0}$ be an integer. We can approximate the Dirichlet polynomial $1+\sum_{m=1}^{N-1} a_{m} m^{-s}$ by a subsequence of partial sums of $\sum_{n=1}^{\infty} a_{n} n^{-s}$ uniformly on the compact set $\{i t: t \in[-1 / 2,1 / 2]\}$. Therefore there exists an integer $M>N$ such that $\left|1-\sum_{m=N}^{M} a_{m} m^{-i t}\right|<1 / 2$ for all $t \in[-1 / 2,1 / 2]$. Hence

$$
\frac{1}{2} \leq \Re\left(\sum_{m=N}^{M} a_{m} m^{-i t}\right) .
$$

We define the convolution product $f(i t)=H_{n_{0}} * \cdots * H_{M}(i t)$, where

$$
h * g(i t)=\frac{1}{2 \pi} \int_{-\pi}^{\pi} h(i x) g(i t-i x) d x .
$$

Note that $f$ is a non-negative $2 i \pi$-periodic function satisfying $f(i t)=0$ for $1 / 2<t \leq \pi$. Hence, multiplying both members of (1) by $f(i t)$ and integrating, we obtain

$$
\frac{1}{2} \leq \sum_{m=N}^{M} \Re\left(a_{m} \int_{-\pi}^{\pi} f(i t) m^{-i t} d t\right) .
$$

By the triangle inequality we have

$$
\frac{1}{2} \leq \sum_{m=N}^{M}\left|a_{m}\right| \cdot|\widehat{f}(m)| .
$$

Moreover, we can calculate

$$
\widehat{f}(m)=\prod_{n=n_{0}}^{M} \frac{\sin \left(\frac{\delta_{n}}{n \log (n)} \log (m)\right)}{\frac{\delta_{n}}{n \log (n)} \log (m)} .
$$

As $\left(\delta_{n}\right)_{n \in \mathbb{N}}$ is a decreasing sequence and the series $\sum_{n \geq 2} \delta_{n} / n \log (n)$ converges, we must have $\lim _{n \rightarrow \infty} \delta_{n}=0$. Therefore, there exists an integer $N$ such that

$$
\frac{\delta_{n_{0}}}{n_{0} \log \left(n_{0}\right)} \log (N)>e \quad \text { and } \quad \delta_{N}<e .
$$


For every $m \in\{N, \ldots, M\}$, we have

$$
\frac{\delta_{n_{0}}}{n_{0} \log \left(n_{0}\right)} \log (m) \geq \frac{\delta_{n_{0}}}{n_{0} \log \left(n_{0}\right)} \log (N)>e
$$

and

$$
\frac{\delta_{m}}{m \log (m)} \log (m)<\delta_{m} \leq \delta_{N}<e .
$$

Then there exists an integer $k \in\left\{n_{0}, \ldots, m-1\right\}$ such that

$$
\frac{\delta_{k}}{k \log (k)} \log (m) \geq e \quad \text { and } \quad \frac{\delta_{k+1}}{(k+1) \log (k+1)} \log (m)<e .
$$

Since the sequence $\delta_{n}$ is decreasing, we also obtain

$$
|\widehat{f}(m)| \leq \prod_{n=n_{0}}^{k} \frac{n \log (n)}{\delta_{n} \log (m)} \leq\left(\frac{k \log (k)}{\delta_{k} \log (m)}\right)^{k+1-n_{0}} \leq\left(\frac{1}{e}\right)^{k+1-n_{0}}
$$

Moreover $(k+1)^{2} \geq(k+1) \log (k+1)>\frac{\delta_{k+1}}{e} \log (m) \geq \varepsilon_{m} \log (m)$ implies $k+1>\sqrt{\varepsilon_{m} \log (m)}$. As a consequence, we find



Since this holds for infinitely many pairs $(N, M)$, the proof is complete.

THEOREM 2.2. Let $\sum_{n=1}^{\infty} a_{n} n^{-s}$ be a Dirichlet series in $\mathcal{W}_{\mathrm{a}}$. Let $\left(b_{n}\right)_{n \in \mathbb{N}}$ be a decreasing sequence such that $\sum_{n=2}^{\infty} b_{n} / n \log (n)<\infty$. Then

$$
\limsup _{n \in \mathbb{N}^{*}} \frac{n\left|a_{n}\right|}{e^{\sqrt{b_{n} \log (n)}}}=\infty \text {. }
$$

Proof. Assume that there exists a real number $M$ such that $\left|a_{n}\right| \leq$ $(M / n) e^{\sqrt{b_{n} \log (n)}}$ for all $n \geq 1$. We set

$$
w_{n}=\max \left(b_{n}, \frac{1}{\sqrt{\log (n)}}\right) \text { and } \varepsilon_{n}=\left(\sqrt{w_{n}}+\sqrt{b_{n}}\right)^{2} .
$$

Note that the sequence $\left(\varepsilon_{n}\right)_{n \in \mathbb{N}}$ is decreasing. Moreover $\sum_{n \geq 2} \frac{\varepsilon_{n}}{n \log (n)}$ converges thanks to the hypothesis on $\left(b_{n}\right)_{n \in \mathbb{N}}$ and the Bertrand criterion. So, by Lemma 2.1, we obtain

$$
\sum_{n=1}^{\infty} \frac{\left|a_{n}\right|}{e^{\sqrt{\varepsilon_{n} \log (n)}}}=\infty
$$

But for every positive integer $A$ we have

$$
\sum_{n=1}^{A} \frac{\left|a_{n}\right|}{e^{\sqrt{\varepsilon_{n} \log (n)}}} \leq M \sum_{n=1}^{A} \frac{1}{n} \frac{1}{e^{\sqrt{\varepsilon_{n} \log (n)}-\sqrt{b_{n} \log (n)}}}=M \sum_{n=1}^{A} \frac{1}{n} \frac{1}{e^{\sqrt{w_{n} \log (n)}}} .
$$


Since $w_{n} \geq 1 / \sqrt{\log (n)}$, we deduce that

$$
\sum_{n=1}^{A} \frac{\left|a_{n}\right|}{e^{\sqrt{\varepsilon_{n} \log (n)}}} \leq M \sum_{n=1}^{A} \frac{1}{n} \frac{1}{e^{\sqrt{\sqrt{\log (n)}}}} .
$$

But the series

$$
\sum_{n \geq 1} \frac{1}{n} \frac{1}{e^{\sqrt{\sqrt{\log (n)}}}}
$$

converges, which contradicts the equality (2) and completes the proof.

Corollary 2.3. Let $\sum_{n=1}^{\infty} a_{n} n^{-s}$ be a Dirichlet series in $\mathcal{W}_{\mathrm{a}}$. Then, for every integer $k$,

$$
\limsup _{n \geq 2} \frac{n\left|a_{n}\right|}{[\log (n)]^{k}}=\infty .
$$

Proof. Let $\left(b_{n}\right)_{n \in \mathbb{N}}$ be the sequence defined by

$$
\forall n \geq 2, \quad b_{n}=\frac{k^{2}[\log (\log (n))]^{2}}{\log (n)} .
$$

This sequence is decreasing (for $n$ large enough) and $\sum_{n=2}^{\infty} b_{n} / n \log (n)$ converges. By Theorem 2.2, we deduce that

$$
\limsup _{n \geq 2} \frac{n\left|a_{n}\right|}{e^{\sqrt{k^{2}(\log (\log (n)))^{2}}}}=\limsup _{n \geq 2} \frac{n\left|a_{n}\right|}{[\log (n)]^{k}}=\infty .
$$

REMARK 2.4. We know by construction that it is possible to build universal Dirichlet series $\sum_{j \geq 1} a_{j} j^{-s}$ satisfying $a_{j}=o\left(j^{-r}\right)$ for any $r<1$ (see [3]). Hence, in contrast to the analytic case [7], we have $\mathcal{W}_{\mathrm{a}} \cap \mathcal{H}^{2} \neq \emptyset$ where $\mathcal{H}^{2}$ is the analogue of $H^{2}(\mathbb{D})$ for Dirichlet series (see for instance [2]).

3. Some properties of $\mathcal{W}_{1}$. We refer the reader to 1.4 for the definition of $\mathcal{W}_{1}$. As mentioned in the introduction, $\mathcal{W}_{1}$ is a dense $G_{\delta}$ subset of $\mathcal{D}_{\mathrm{a}}\left(\mathbb{C}_{+}\right)$. Moreover, note that such series have abscissas of convergence and absolute convergence both equal to 0 . To see that, just take as $K$ any singleton $\left\{z_{0}\right\}$ with $\Re\left(z_{0}\right)<0$. But what happens on the imaginary axis?

Notation 3.1. Let $\left(f_{j}\right)_{j \in \mathbb{N}}$ be an enumeration of the Dirichlet polynomials with coefficients in $\mathbb{Q}+i \mathbb{Q}$. We also consider a family $\left\{K_{\varrho}\right\}_{\varrho \in \mathbb{N}}$ of admissible compact sets of $\mathbb{C}_{-}$such that for every admissible compact set $K \subset \mathbb{C}_{-}$, there exists a nonnegative integer $\varrho_{0}$ with $K \subset K_{\varrho_{0}}$ (see [5]).

Finally, for a Dirichlet polynomial $P(s)=\sum_{n=1}^{n_{0}} a_{n} n^{-s}$ with $a_{n_{0}} \neq 0$ the degree is defined to be $\operatorname{deg}(P)=n_{0}$.

According to a suggestion of J.-P. Kahane, we easily obtain the following proposition (see [8, Proposition 3.2]). 
Proposition 3.2. Let $f$ be a Dirichlet series in $\mathcal{D}_{\mathrm{a}}\left(\mathbb{C}_{+}\right)$. Then there exist $g_{1}$ and $g_{2}$ in $\mathcal{W}_{1}$ such that $f=g_{1}+g_{2}$.

Here we give another version of this proposition with additional conditions on the growth of coefficients. First of all we need a more precise version of Mergelyan's theorem for Dirichlet series.

Lemma 3.3. Let $K$ be an admissible compact set included in $\mathbb{C}_{-}$. Let also $g$ be a continuous function on $K$, analytic in the interior of $K$, and $\varepsilon, \sigma$ be strictly positive real numbers. Then there exists a Dirichlet polynomial $h(s)=\sum_{n \geq 1} h_{n} n^{-s}$ satisfying

$$
\left\{\begin{array}{l}
\sup _{z \in K}|h(z)-g(z)|<\varepsilon \\
\|h\|_{\sigma}<\varepsilon \\
\forall n \in \mathbb{N}^{*}, \quad n\left|h_{n}\right|<\varepsilon
\end{array}\right.
$$

Proof. We use the notations of Lemma 2 from [3] in the special case $f=0 \in \mathcal{D}_{\mathrm{a}}\left(\mathbb{C}_{+}\right)$. We write $K=K_{1} \cup \cdots \cup K_{d}$. Then there exist positive real numbers $\sigma_{1}<\cdots<\sigma_{d}$ and natural numbers $n_{1}<m_{1}<n_{2}<m_{2}<\cdots<$ $n_{d}<m_{d}$ such that the Dirichlet polynomial

$$
h(s)=\sum_{l=1}^{d} \sum_{j=n_{l}+1}^{m_{l}} b_{j}^{(l)} j^{-\sigma_{l}} j^{-s}=\sum_{j=n_{1}+1}^{\infty} h_{j} j^{-s}
$$

satisfies $\sup _{z \in K}|h(z)-g(z)|<\varepsilon$ and $\|h\|_{\sigma}<\varepsilon$. Notice that $n_{1}$ can be chosen arbitrarily large ([1]). Moreover, from the result of [1], the moduli of the complex numbers $b_{j}^{(l)}$ are upper bounded by 1 . Therefore, we obtain, for all $j \in \mathbb{N}^{*}$,

$$
\left|j h_{j}\right| \leq j\left|b_{j}^{(l)} j^{-\sigma_{l}}\right| \leq j^{-\sigma_{1}+1} \leq n_{1}^{-\sigma_{1}+1}
$$

We just have to choose an integer $n_{1}$ satisfying $n_{1}^{-\sigma_{1}+1}<\varepsilon$ to complete the proof, which is possible because the compact set does not intersect the imaginary axis and therefore we can choose $\sigma_{1}>1$ and have $\sigma_{1}+K_{1} \subset\{s \in$ $\mathbb{C}: 1 / 2<\Re(s)<1\}$.

REMARK 3.4. Note that we need the condition $K \subset \mathbb{C}_{-}$to obtain a control on the coefficients $n\left|h_{n}\right|$ from the previous lemma, which is not possible in the case of the set $\mathcal{W}_{a}$.

Corollary 3.5. Let $K$ be an admissible compact set included in $\mathbb{C}_{-}$. Let also $g$ be a continuous function on $K$, analytic in the interior of $K, \varepsilon, \sigma$ be strictly positive real numbers, and $\lambda$ be a strictly positive integer. Then 
there exists a Dirichlet polynomial $h(s)=\sum_{n \geq 1} h_{n} n^{-s}$ satisfying

$$
\left\{\begin{array}{l}
\sup _{s \in K}\left|g(s)-\lambda^{-s} h(s)\right|<\varepsilon, \\
\left\|\lambda^{-s} h(s)\right\|_{\sigma}<\varepsilon \\
\forall n \in \mathbb{N}^{*}, \quad n \lambda\left|h_{n}\right|<\varepsilon
\end{array}\right.
$$

Proof. Using the notations of Lemma 3.3, for each $\varepsilon_{1}$ there exists a Dirichlet polynomial $h$ such that $\sup _{s \in K}\left|g(s) \lambda^{s}-h(s)\right|<\varepsilon_{1},\|h\|_{\sigma}<\varepsilon_{1}$ and $n\left|h_{n}\right|<\varepsilon_{1}$. Therefore,

$$
\left(\inf _{s \in K}\left|\lambda^{s}\right|\right)\left(\sup _{s \in K}\left|g(s)-\lambda^{-s} h(s)\right|\right)<\varepsilon_{1} .
$$

We just have to choose $\varepsilon_{1}$ such that $\max \left(\varepsilon_{1} / \inf _{s \in K}\left|\lambda^{s}\right|, \lambda \varepsilon_{1}\right)<\varepsilon$.

We can now use the main ideas from [7] to obtain the following result.

Theorem 3.6. Let $f=\sum_{n \geq 1} d_{n} n^{-s}$ be a Dirichlet series in $\mathcal{D}_{\mathrm{a}}\left(\mathbb{C}_{+}\right)$. There exist $g_{1}=\sum_{n \geq 1} a_{n} n^{-s}$ and $g_{2}=\sum_{n \geq 1} b_{n} n^{-s}$ in $\mathcal{W}_{1}$ such that $f=$ $g_{1}+g_{2}$ on $\mathbb{C}_{+}$with

$$
\limsup _{n \in \mathbb{N}} n\left|a_{n}\right|=\limsup _{n \in \mathbb{N}} n\left|b_{n}\right|=\limsup _{n \in \mathbb{N}} n\left|d_{n}\right| .
$$

Proof. First we study the case $\lim \sup _{n \in \mathbb{N}} n\left|d_{n}\right|=\infty$. By Proposition 3.2 , there exist $g_{1}$ and $g_{2}$ in $\mathcal{W}_{\mathrm{a}} \subset \mathcal{W}_{1}$ satisfying $f=g_{1}+g_{2}$. The conclusion is given by Corollary 2.3 .

Case $\lim \sup _{n \in \mathbb{N}} n\left|d_{n}\right|<\infty$. We have a countable family $\left(K_{\varrho_{i}}, f_{j_{i}}\right)$. Fix $\lambda_{1}=1$. By Corollary 3.5, there exists a Dirichlet polynomial $P_{1}(s)=$ $\sum_{n \geq 1} p_{1, n} n^{-s}$ such that

$$
\begin{gathered}
\sup _{s \in K_{\varrho_{1}}}\left|f_{j_{1}}(s)-\lambda_{1}^{-s} P_{1}(s)\right|<1, \quad\left\|\lambda_{1}^{-s} P_{1}(s)\right\|_{\sigma_{1}}<1, \\
\forall n \in \mathbb{N}, \quad n \lambda_{1}\left|p_{1, n}\right|<1 .
\end{gathered}
$$

We choose $\mu_{1}>\lambda_{1}+\operatorname{deg}\left(P_{1}\right) \geq \lambda_{1}$ such that

$$
\limsup _{n \in \mathbb{N}} n\left|d_{n}\right|-\max \left\{l\left|d_{l}\right|: \mu_{1}>l>\lambda_{1}+\operatorname{deg}\left(P_{1}\right)\right\}<1 .
$$

Then define the Dirichlet polynomial

$$
R_{1}(s)=\sum_{n=\lambda_{1}}^{\mu_{1}-1} d_{n} n^{-s}-\lambda_{1}^{-s} P_{1}(s) .
$$

By Corollary 3.5, there exists a Dirichlet polynomial $Q_{1}(s)=\sum_{n \geq 1} q_{1, n} n^{-s}$ satisfying

$$
\begin{gathered}
\sup _{s \in K_{\varrho_{1}}}\left|f_{j_{1}}(s)-R_{1}(s)-\mu_{1}^{-s} Q_{1}(s)\right|<1, \quad\left\|\mu_{1}^{-s} Q_{1}\right\|_{\sigma_{1}}<1 \\
\forall n \in \mathbb{N}, \quad n \mu_{1}\left|q_{1, n}\right|<1 .
\end{gathered}
$$


Let $\lambda_{2}$ be an integer satisfying $\lambda_{2}>\mu_{1}+\operatorname{deg}\left(Q_{1}\right) \geq \mu_{1}>\lambda_{1}$ and

$$
\limsup _{n \in \mathbb{N}} n\left|d_{n}\right|-\max \left\{l\left|d_{l}\right|: \lambda_{2}>l>\mu_{1}+\operatorname{deg}\left(Q_{1}\right)\right\}<1 \text {. }
$$

We set

$$
F_{1}(s)=\sum_{n=\mu_{1}}^{\lambda_{2}-1} d_{n} n^{-s}-\mu_{1}^{-s} Q_{1}(s) .
$$

We construct the sequences $\tilde{\lambda}=\left(\lambda_{k}\right)_{k \geq 1}$ and $\widetilde{\mu}=\left(\mu_{k}\right)_{k \geq 1}$ step by step. Assume that we have

$$
1=\lambda_{1}<\mu_{1}<\lambda_{2}<\mu_{2}<\cdots<\lambda_{k-1}<\mu_{k-1}<\lambda_{k}
$$

and that the Dirichlet polynomials $P_{i}, Q_{i}, R_{i}$ and $F_{i}$ are constructed for $i=$ $1, \ldots, k-1$. By Corollary 3.5, there exists a Dirichlet polynomial $P_{k}(s)=$ $\sum_{n \geq 1} p_{k, n} n^{-s}$ such that

$$
\begin{gathered}
\sup _{s \in K_{\varrho_{k}}}\left|f_{j_{k}}(s)-\sum_{j=1}^{k-1}\left(\lambda_{j}^{-s} P_{j}(s)+F_{j}(s)\right)-\lambda_{k}^{-s} P_{k}(s)\right|<1 / k^{2}, \\
\left\|\lambda_{k}^{-s} P_{k}(s)\right\|_{\sigma_{k}} \leq 1 / k^{2} \text { and } \forall n \in \mathbb{N}, \quad n \lambda_{k}\left|p_{k, n}\right|<1 / k^{2} .
\end{gathered}
$$

Further, we determine $\mu_{k}>\lambda_{k}+\operatorname{deg}\left(P_{k}\right)$ such that

$$
\limsup _{n \in \mathbb{N}} n\left|d_{n}\right|-\max \left\{l\left|d_{l}\right|: \mu_{k}>l>\lambda_{k}+\operatorname{deg}\left(P_{k}\right)\right\}<1 / k^{2}
$$

and we set

$$
R_{k}(s)=\sum_{n=\lambda_{k}}^{\mu_{k}-1} d_{n} n^{-s}-\lambda_{k}^{-s} R_{k}(s) .
$$

By Corollary 3.5, there exists a Dirichlet polynomial $Q_{k}(s)=\sum_{n \geq 1} q_{k, n} n^{-s}$ satisfying

$$
\begin{gathered}
\sup _{s \in K_{\varrho_{k}}}\left|f_{j_{k}}(s)-\sum_{j=1}^{k-1}\left(\mu_{j}^{-s} Q_{j}(s)+R_{j}(s)\right)-R_{k}(s)-\mu_{k}^{-s} Q_{k}(s)\right|<1 / k^{2}, \\
\left\|\mu_{k}^{-s} Q_{k}\right\|_{\sigma_{k}}<1 / k^{2} \quad \text { and } \quad \forall n \in \mathbb{N}, \quad n \mu_{k}\left|q_{k, n}\right|<1 / k^{2} .
\end{gathered}
$$

Let $\lambda_{k+1}$ be an integer satisfying $\lambda_{k+1}>\mu_{k}+\operatorname{deg}\left(Q_{k}\right) \geq \mu_{k}>\lambda_{k}$ and

$$
\limsup _{n \in \mathbb{N}} n\left|d_{n}\right|-\max \left\{l\left|d_{l}\right|: \lambda_{k+1}>l>\mu_{k}+\operatorname{deg}\left(Q_{k}\right)\right\}<1 / k^{2} .
$$

We set

$$
F_{k}(s)=\sum_{n=\mu_{k}}^{\lambda_{k+1}-1} d_{n} n^{-s}-\mu_{k}^{-s} Q_{k}(s) .
$$

Since $\left\|\lambda_{k}^{-s} P_{k}(s)\right\|_{\sigma_{k}}<1 / k^{2}$ and $\left\|\mu_{k}^{-s} Q_{k}(s)\right\|_{\sigma_{k}}<1 / k^{2}$, the two Dirichlet series $\sum_{k \geq 1} \lambda_{k}^{-s} P_{k}(s)$ and $\sum_{k \geq 1} \mu_{k}^{-s} Q_{k}(s)$ are in $\mathcal{D}_{\mathrm{a}}\left(\mathbb{C}_{+}\right)$(the terms with 
index $n$ appear only once, moreover the sequence $\widetilde{\sigma}$ is decreasing, therefore the associated seminorms increase). As the Dirichlet series $f$ is absolutely convergent on $\mathbb{C}_{+}$, so does

$$
\sum_{k=1}^{\infty} \sum_{n=\mu_{k}}^{\lambda_{k+1}-1} d_{n} n^{-s}
$$

(by construction the sums are disjoint). Therefore the Dirichlet series

$$
\sum_{k \geq 1} \lambda_{k}^{-s} P_{k}(s)+\sum_{k=1}^{\infty} \sum_{n=\mu_{k}}^{\lambda_{k+1}-1} d_{n} n^{-s}-\sum_{k \geq 1} \mu_{k}^{-s} Q_{k}(s)=\sum_{k \geq 1}\left(\lambda_{k}^{-s} P_{k}(s)+F_{k}(s)\right)
$$

is an element of $\mathcal{D}_{a}\left(\mathbb{C}_{+}\right)$. We denote by $\sum_{k \geq 1} a_{n} n^{-s}$ the development of this function. For $N=\lambda_{k}+\operatorname{deg}\left(P_{k}\right)$, we have

$$
\sum_{n=1}^{N} a_{n} n^{-s}=\lambda_{1}^{-s} P_{1}(s)+F_{1}(s)+\cdots+\lambda_{k-1}^{-s} P_{k-1}(s)+F_{k-1}(s)+\lambda_{k}^{-s} P_{k}(s) .
$$

Similarly, we define another Dirichlet series in $\mathcal{D}_{\mathrm{a}}\left(\mathbb{C}_{+}\right)$,

$$
-\sum_{k \geq 1} \lambda_{k}^{-s} P_{k}(s)+\sum_{k=1}^{\infty} \sum_{n=\lambda_{k}}^{\mu_{k}-1} d_{n} n^{-s}+\sum_{k \geq 1} \mu_{k}^{-s} Q_{k}(s)=\sum_{k \geq 1}\left(R_{k}(s)+\mu_{k}^{-s} Q_{k}(s)\right),
$$

and we denote by $\sum_{n \geq 1} b_{n} n^{-s}$ the development of this function. By construction, we have the relation

$$
\forall n \geq 1, \quad d_{n}=a_{n}+b_{n} .
$$

Moreover, by Corollary 3.5, we know that all the coefficients which appear in the decomposition of $\lambda_{k}^{-s} P_{k}(s)=\sum_{n \geq 1} p_{k, n}\left(\lambda_{k} n\right)^{-s}$ and $\mu_{k}^{-s} Q_{k}(s)=$ $\sum_{n \geq 1} q_{k, n}\left(\mu_{k} n\right)^{-s}$ (denoted by $\left(r_{k, n}\right)$ and $\left(s_{k, n}\right)$ respectively) satisfy

$$
\lambda_{k} n\left|r_{k, \lambda_{k} n}\right|=\lambda_{k} n\left|p_{k, n}\right| \leq 1 / k^{2}, \quad \mu_{k} n\left|s_{k, \mu_{k} n}\right|=\mu_{k} n\left|q_{k, n}\right| \leq 1 / k^{2} .
$$

Hence, the coefficients $t_{n}$ of the series $\sum_{k \geq 1} \lambda_{k}^{-s} P_{k}(s)-\sum_{k \geq 1} \mu_{k}^{-s} Q_{k}(s)$ satisfy $n\left|t_{n}\right| \rightarrow 0$. Therefore, we have the following estimates:

$$
\limsup _{n \in \mathbb{N}} n\left|a_{n}\right| \leq \limsup _{n \in \mathbb{N}} n\left|d_{n}\right| \quad \text { and } \quad \limsup _{n \in \mathbb{N}} n\left|b_{n}\right| \leq \limsup _{n \in \mathbb{N}} n\left|d_{n}\right| .
$$

Further, for $l$ satisfying $\mu_{k}+\operatorname{deg}\left(Q_{k}\right)<l<\lambda_{k+1}$, we have $d_{l}=a_{l}$ and

$$
\limsup _{n \in \mathbb{N}} n\left|d_{n}\right|-\max \left\{l\left|d_{l}\right|: \mu_{k}+\operatorname{deg}\left(Q_{k}\right)<l<\lambda_{k+1}\right\}<1 / k^{2} .
$$

As an easy consequence, we have $\lim \sup _{n \in \mathbb{N}} n\left|d_{n}\right|=\lim \sup _{n \in \mathbb{N}} n\left|a_{n}\right|$. Similarly, $\lim \sup _{n \in \mathbb{N}} n\left|d_{n}\right|=\lim \sup _{n \in \mathbb{N}} n\left|b_{n}\right|$. To conclude the proof, we have to show that the two elements $\sum_{n \geq 1} a_{n} n^{-s}$ and $\sum_{n \geq 1} b_{n} n^{-s}$ belong to $\mathcal{W}_{1}$. Let $K$ be an admissible compact set in $\mathbb{C}_{-}$and $h$ be a continuous function 
on $K$, analytic in the interior of $K$. Let $\varepsilon>0$ and $v$ be a natural number. We shall find $N>v$ such that

$$
\sup _{s \in K}\left|h(s)-\sum_{n=1}^{N} a_{n} n^{-s}\right|<\varepsilon .
$$

There exists a sequence $f_{\lambda}(\lambda=1,2, \ldots)$ such that

$$
\sup _{s \in K}\left|h(s)-f_{\lambda}\right|<\varepsilon / 2 .
$$

Moreover, there exists a sequence $\left(\varrho_{p}\right)_{p \geq 0}$ such that $K \subset K_{\varrho_{p}}$ and we can consider the set $\left\{\left(K_{\varrho}, f_{\lambda}+q\right): q \in \mathbb{Q}, 0<q<\varepsilon / 4\right\}$ to conclude as in Theorem 5.1 of [7].

COROLlary 3.7. We have the strict inclusion $\mathcal{W}_{\mathrm{a}} \varsubsetneqq \mathcal{W}_{1}$.

Proof. We just have to apply Theorem 3.6 with $d_{n} \equiv 1 / n$ or $d_{n} \equiv 0$. Corollary 2.3 implies that $g_{1}$ and $g_{2}$ cannot be in $\mathcal{W}_{\mathrm{a}}$.

In the universal set $\mathcal{W}_{1}$, a natural problem is the existence of universal series which converge on the imaginary axis and are continuous. In the case of Taylor series this is proved by the study of universality on the Banach space $A(\mathbb{D})$ of analytic functions on $\mathbb{D}$, continuous on the torus $\mathbb{T}$ (see [7]). In the next section we also give a positive answer in the Dirichlet case, by introducing universal series in the Wiener-Dirichlet algebra.

4. Universality in the Wiener-Dirichlet algebra. The classical Wiener algebra of absolutely convergent Taylor series in one variable is the set of functions $f(z)=\sum_{n=0}^{\infty} a_{n} z^{n}$ such that $\sum_{n=0}^{\infty}\left|a_{n}\right|<\infty$. Similarly, we can define the Wiener-Dirichlet algebra, denoted in the following by $\mathcal{D}_{\mathrm{w}}$. A Dirichlet series $f(s)=\sum_{n \geq 1} a_{n} n^{-s}$ belongs to the WienerDirichlet algebra $\mathcal{D}_{\mathrm{w}}$ if $\|f\|=\sum_{n=1}^{\infty}\left|a_{n}\right|<\infty$. Endowed with this norm, $\mathcal{D}_{\mathrm{w}}$ is obviously a Banach algebra. These two algebras are not completely similar. Indeed, it is well-known that the spectrum of the Wiener algebra is $\overline{\mathbb{D}}$, the closed unit disk. For the Wiener-Dirichlet algebra, using the classical Bohr's viewpoint [4], we can prove that its spectrum is $\overline{\mathbb{D}}^{\infty}$. Finally, we can easily see that the Wiener-Dirichlet algebra is a subset of $\mathcal{D}_{\mathrm{a}}\left(\mathbb{C}_{+}\right)$.

Definition 4.1. We denote by $\mathcal{U}_{\mathrm{wd}}$ the set of all Dirichlet series $h \in \mathcal{D}_{\mathrm{w}}$ satisfying: for every admissible compact set $K \subset \mathbb{C}_{-}$and every function $g$, continuous on $K$ and analytic in $K_{\circ}$, there exists a sequence $\left(\lambda_{n}\right)_{n \geq 0}$ of natural numbers such that

$$
\sup _{z \in K}\left|S_{\lambda_{n}}(h)(z)-g(z)\right| \underset{n \rightarrow \infty}{\longrightarrow} 0 .
$$

TheOREM 4.2. $\mathcal{U}_{\mathrm{wd}}$ is a dense $G_{\delta}$ in $\mathcal{D}_{\mathrm{w}}$. 
In [9] a short proof of Theorem 4.2 has been given independently and simultaneously. We only give the steps of our proof. Using the notations of 3.1 we observe that the family of Dirichlet polynomials $\left(f_{j}\right)_{j \in \mathbb{N}}$ is dense in $\mathcal{D}_{\mathrm{w}}$. Using again the notation of 3.1 we recall that $K_{\varrho}, \varrho=1,2, \ldots$, are admissible compact sets in $\mathbb{C}_{-}$absorbing all other such sets. For positive integers $\varrho, j, n, s$ we define

$$
\mathcal{O}_{\mathrm{w}}(\varrho, j, s, n)=\left\{g \in \mathcal{D}_{\mathrm{w}}: \sup _{z \in K_{\varrho}}\left|S_{n}(g)(z)-f_{j}(z)\right|<1 / s\right\}
$$

Then we show

$$
\mathcal{U}_{\mathrm{wd}}=\bigcap_{\varrho=1}^{\infty} \bigcap_{j=0}^{\infty} \bigcap_{s=1}^{\infty} \bigcup_{n=1}^{\infty} \mathcal{O}_{\mathrm{w}}(\varrho, j, s, n) .
$$

We also show that the set $\mathcal{O}_{\mathrm{w}}(\varrho, j, s, n)$ is open in $\mathcal{D}_{\mathrm{w}}$. Finally, using a version of Lemma 2 from [3] (see also [1]), which allows $\sigma=0$ when the compact set $K$ is included in $\mathbb{C}_{-}$, we prove that $\bigcup_{n=1}^{\infty} \mathcal{O}_{\mathrm{w}}(\varrho, j, s, n)$ is dense in $\mathcal{D}_{\mathrm{w}}$. Then the completeness of $\mathcal{D}_{\mathrm{w}}$ allows the use of Baire's category theorem, which yields Theorem 4.2.

As a consequence of our main result, we obtain information on the universal set $\mathcal{W}_{1}$. Moreover, we specify the strict inclusion $\mathcal{W}_{\text {a }} \varsubsetneqq \mathcal{W}_{1}$.

Corollary 4.3. We have the inclusion $\mathcal{W}_{\mathrm{a}} \subset \mathcal{W}_{1} \cap \mathcal{U}_{\mathrm{wd}}^{\mathrm{c}}$.

Proof. Every series from $\mathcal{W}_{a}$ converges nowhere on the imaginary axis. Therefore $\mathcal{W}_{\mathrm{a}} \cap \mathcal{U}_{\mathrm{wd}}=\emptyset$.

\section{References}

[1] B. Bagchi, A joint universality theorem for Dirichlet L-functions, Math. Z. 181 (1982), 319-334.

[2] F. Bayart, Hardy spaces of Dirichlet series and their composition operators, Monatsh. Math. 136 (2002) 3, 203-236.

[3] -, Topological and algebraic genericity of divergence and universality, Studia Math. 167 (2005), 161-181.

[4] F. Bayart, C. Finet, D. Li and H. Queffélec, Composition operators on the WienerDirichlet algebra, J. Operator Theory, to appear.

[5] O. Demanze and A. Mouze, Universal approximation theorem for Dirichlet series, Int. J. Math. Math. Sci. 2006, art. ID 37014, 11 pp.

[6] J.-P. Kahane et V. Nestoridis, Séries de Taylor et séries trigonométriques universelles au sens de Menchoff, J. Math. Pures Appl. 79 (2000), 855-862.

[7] A. Melas, V. Nestoridis and I. Papadoperakis, Growth of coefficients of universal Taylor series and comparison of two classes of functions, J. Anal. Math. 73 (1997) 187-202.

[8] V. Nestoridis, Universal Taylor series, Ann. Inst. Fourier (Grenoble) 46 (1996), 1293-1306.

[9] V. Nestoridis and C. Papadimitropoulos, Abstract theory of universal series and an application to Dirichlet series, C. R. Math. Acad. Sci. Paris 341 (2005), 539-543. 
[10] L. Tomm and R. Trautner, A universal power series for approximation of measurable functions, Analysis 2 (1982), 1-6.

Olivier Demanze

E-mail: demanze-o.math@laposte.net
Augustin Mouze

Laboratoire de Mathématiques, UMR 8524

École Centrale de Lille

Cité Scientifique

59650 Villeneuve d'Ascq, France

E-mail: Augustin.Mouze@math.univ-lille1.fr

Received 25.5.2006

and in final form 24.1.2007 